\title{
Reconstruction of protein structures from a vectorial representation
}

\author{
Markus Porto, ${ }^{1,2}$ Ugo Bastolla, ${ }^{3}$ H. Eduardo Roman, ${ }^{4}$ and Michele Vendruscolo ${ }^{5}$ \\ ${ }^{1}$ Max-Planck-Institut für Physik komplexer Systeme, Nöthnitzer Straße 38, 01187 Dresden, Germany \\ ${ }^{2}$ Institut für Theoretische Physik, Technische Universität Dresden, 01062 Dresden, Germany \\ ${ }^{3}$ Centro de Astrobiología (INTA-CSIC), 28850 Torrejón de Ardoz, Spain \\ ${ }^{4}$ Dipartimento di Fisica and INFN, Università di Milano, Via Celoria 16, 20133 Milano, Italy \\ ${ }^{5}$ Department of Chemistry, University of Cambridge, Lensfield Road, Cambridge CB2 1EW, UK
}

(Dated: September 5, 2003, revised January 7, 2004)

\begin{abstract}
We show that the contact map of the native structure of globular proteins can be reconstructed starting from the sole knowledge of the contact map's principal eigenvector, and present an exact algorithm for this purpose. Our algorithm yields a unique contact map for all 221 globular structures of PDBselect25 of length $N \leq 120$. We also show that the reconstructed contact maps allow in turn for the accurate reconstruction of the three-dimensional structure. These results indicate that the reduced vectorial representation provided by the principal eigenvector of the contact map is equivalent to the protein structure itself. This representation is expected to provide a useful tool in bioinformatics algorithms for protein structure comparison and alignment, as well as a promising intermediate step towards protein structure prediction.
\end{abstract}

PACS numbers: 87.14.Ee, 87.15.Cc, 87.15.Aa

Introduction. - Despite several decades of intense research, the reliable prediction of the native state of a protein from its sequence of amino acids is still a formidable challenge 1]. Every two years the state of the art is assessed by the CASP experiment [2]. In the most detailed predictions, the list of the Cartesian coordinates of all the atoms of the protein molecule are provided. Since the structure of a protein can be also represented as a contact map [3], lower-resolution predictions can be limited to the determination of inter-residue contacts [4].

Ideally, one would like to predict the structure of a protein using the representation that is encoded in the most straightforward way in the sequence of amino acids. Due to the vectorial nature of the sequence, one may guess that the simplest representation to predict should be vectorial as well. In this Letter, we show that the principal eigenvector $(\mathrm{PE})$ of the contact map $(\mathrm{CM})$ of the native structure is equivalent to the CM itself, and therefore provides a very promising vectorial representation of protein structures. This vectorial representation is also expected to improve bioinformatics algorithms for protein structure alignment as well as alignment of protein sequence with a database of structures (fold recognition).

The PE of the CM has already been used as an indicator of protein topology, in particular as a mean of identifying structural domains 5 and clusters of amino acids with special structural significance [6, 7]. Here, we present an exact algorithm to reconstruct a CM from the knowledge of its PE (cf. Fig. (1). This step is analogous to that of reconstructing the three-dimensional protein structure based on the CM of the structure [8]. For the proteins that we studied, the PE is sufficient to reconstruct uniquely a CM. This means that the information about all other eigenvectors and eigenvalues of a $\mathrm{CM}$ is contained in the PE, and therefore it is equivalent to represent a protein structure either by the $\mathrm{CM}$ or the $\mathrm{PE}$ of the CM. This result is likely due to the binary nature of the entries of the CM and to the fact that the topology of the protein chain imposes significant constraints on the non-zero entries $[$ ].

Contact maps and their principal eigenvectors. - The contact map $\mathbf{C}$ of a protein structure is a binary symmetric matrix, with elements $C_{i j}=1$ if amino acids at positions $i$ and $j$ are in contact, and 0 otherwise [3, 8]. Two residues are defined to be in contact if at least one pair of heavy atoms, one belonging to each amino acid, are less than $4.5 \AA$ apart. Other contact definitions exist in the literature, for instance based on a distance threshold on the $\mathrm{C}_{\alpha}$ atoms [8], but the algorithm presented below does not depend on the detailed contact condition. Additionally, only residues separated by at least three positions along the sequence are considered in contact, so that $C_{i j}=0$ if $|i-j|<3$. In such a way, trivial short range contacts are not taken into account.

In what follows, $\lambda$ will denote the principal (i.e., largest) eigenvalue of $\mathbf{C}$ [10], and $\mathbf{v}$ the corresponding $\mathrm{PE}$. Since $\mathbf{C}$ is a real symmetric matrix, its eigenvalues are real. The principal eigenvalue $\lambda$ has a value between the average number of contacts per residue, $\left\langle\sum_{j} C_{i j}\right\rangle_{i}$, and the maximal number of contacts of any given residue, $\max _{i}\left(\sum_{j} C_{i j}\right)$ [1]. The non-zero components of $\mathbf{v}$ have all the same sign, which we choose to be positive.

The PE maximizes the quadratic form $\sum_{i j} C_{i j} v_{i} v_{j}$ with the constraint $\sum_{i} v_{i}^{2}=1$. In this sense, $v_{i}$ can be interpreted as the effective connectivity of position $i$, since positions with large $v_{i}$ are in contact with as many as possible positions $j$ with large $v_{j}$. As we discuss below, one can show that if the CM represents a single connected graph, all the structural information is actually contained in its PE. For proteins consisting of several distinct domains, the $\mathrm{CM}$ becomes a block matrix, and the PE contains information only on the largest block 

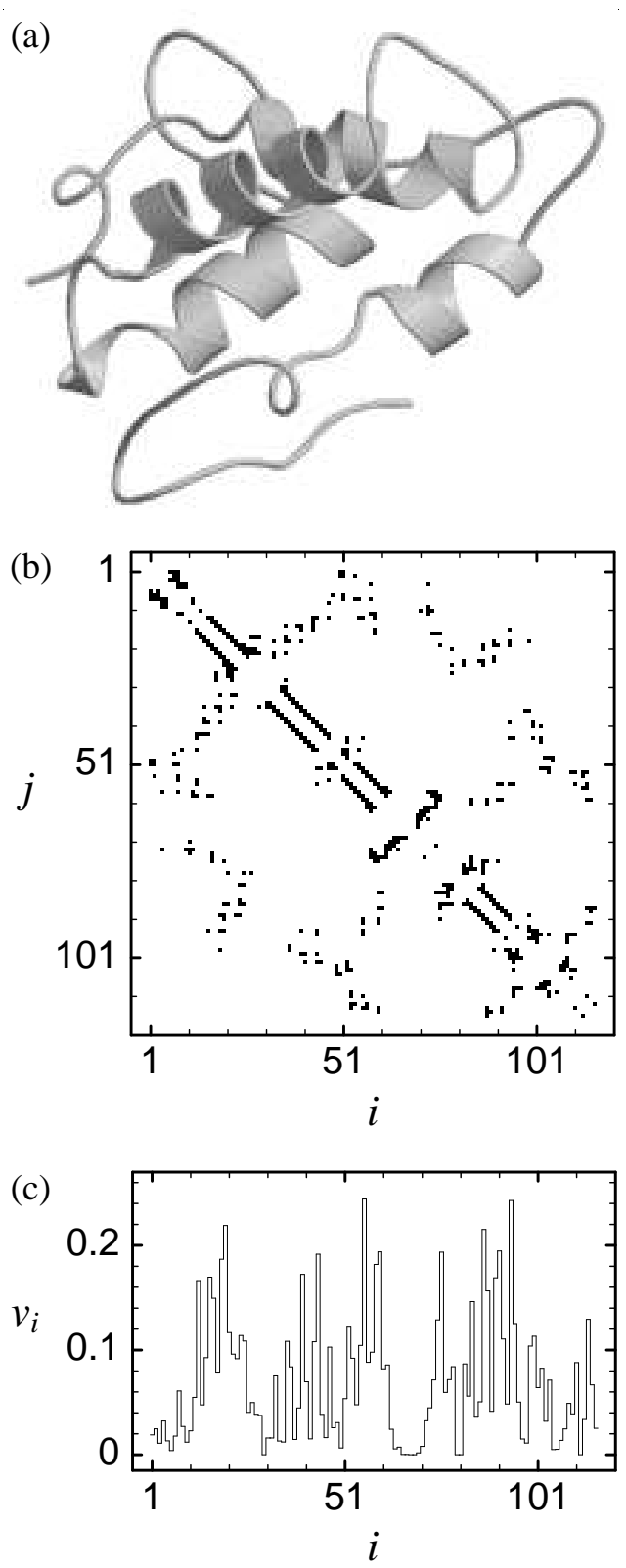

FIG. 1: The protein Amylase/Serine protease inhibitor with PDB id. 1bea. Shown are: (a) The three-dimensional structure (drawn using MolScript and Raster3D), (b) the CM C (black means $C_{i j}=1$ and white means $C_{i j}=0$ ), and (c) the $\mathrm{PE} \mathbf{v}$. The reduction from (a) to (b) is done by a distance threshold, and the one from (b) to (c) is by a diagonalization. The reconstruction from (c) to (b) is described in this Letter, whereas the one from (b) to (a) is described in Ref. [8].

(domain).

Reconstructing a contact map from its principal eigenvector. - The reconstruction algorithm is based on imposing that the matrix $\mathbf{C}$, applied to $\mathbf{v}$, fulfills the secular equation $\mathbf{C v}=\lambda \mathbf{v}$. At first glance, this equation does not seem to be sufficient to determine the $\mathrm{CM}$, as there are infinitely many matrices $\mathbf{M} \neq \mathbf{C}$ that fulfill $\mathbf{M v}=\lambda \mathbf{v}$. However, additional constraints can be imposed using the fact that the elements of $\mathbf{C}$ assume only the values 0 and 1 , and that consequently $\lambda$ as well as all non-zero components of $\mathbf{v}$ are positive. The central idea is to exploit these additional constraints, as they allow to apply a 'greedy' scheme to search for possible solutions. We first discuss the algorithm in the hypothesis that all components of the $\mathbf{v}$ and the corresponding principal eigenvalue $\lambda$ are known. We will show later that, for CMs of protein folds, it is possible to deduce $\lambda$ from the components of the $\mathrm{PE}$, so that only this quantity has to be known. The algorithm proceeds according to the following steps:

(i) Elements $C_{i j}$ with $|i-j|<3$ are set to 0 . The remaining elements of $\mathbf{C}$ are marked as 'unknown.'

(ii) For all positions $i$ for which the PE vanishes, i.e. $v_{i}=0$, all 'unknown' entries in the $i$-th line and the $i$ th column of $\mathbf{C}$ are set to 0 , i.e. $C_{i j}=C_{j i}=0$ for all $j$ (concerning 'unknown' elements $C_{i j}$ for which both $v_{i}$ and $v_{j}$ vanish see below).

(iii) The non-zero components of $\mathbf{v}$ are sorted and treated recursively in increasing order, starting with the smallest value. Let $i$ be the position presently examined, with the aim to determine the $i$-th line and $i$-th column of $\mathbf{C}$. Some elements $C_{i j}=C_{j i}$ with $j \in \mathcal{J}=\left\{j_{1}, j_{2}, \ldots\right\}$ are already known, whereas some other elements $C_{i k}=C_{k i}$ with $k \in \mathcal{K}=\left\{k_{1}, k_{2}, \ldots\right\}$ are still 'unknown.' To evaluate the latter, we calculate the sum $\sum_{j \in \mathcal{J}} C_{i j} v_{j}$ of the known elements $\mathcal{J}$, and consider three different possibilities:

(iii/1) The sum $\sum_{j \in \mathcal{J}} C_{i j} v_{j}$ is equal to $\lambda v_{i}$ up to the chosen precision $\epsilon$, i.e. $\left|\sum_{j \in \mathcal{J}} C_{i j} v_{j}-\lambda v_{i}\right|<\epsilon$. Then one solution for the $i$-th line and the $i$-th column of $\mathbf{C}$ has been found, since the $i$-th line of the secular equation can be fulfilled by setting to 0 all 'unknown' elements in the $i$-th line and the $i$-th column of $\mathbf{C}, C_{i k}=C_{k i}=0$ for $k \in \mathcal{K}$, and the recursion returns with success.

(iii/2) Otherwise, if either $\sum_{j \in \mathcal{J}} C_{i j} v_{j}>\lambda v_{i}$, meaning numerically that $\sum_{j \in \mathcal{J}} C_{i j} v_{j}-\lambda v_{i} \geq \epsilon$, or if the set of 'unknown' entries $\mathcal{K}$ is empty, the $i$-th line of the secular equation cannot be fulfilled with the present set $\mathcal{J}$, the recursion is in a dead end and returns with failure.

(iii/3) Finally, in the remaining cases, the 'unknown' elements in the non-empty set $\mathcal{K}$ have to be further processed. To eliminate candidates which lead to a dead end, the set of elements $\mathcal{K}$ is sorted by the values $v_{k}$ in decreasing order. Starting with the element $k \in \mathcal{K}$ which has the largest value $v_{k}$, the sum $\sum_{j \in \mathcal{J}} C_{i j} v_{j}+v_{k}$ is calculated. There are two possibilities: (a) If $\sum_{j \in \mathcal{J}} C_{i j} v_{j}+v_{k}>\lambda v_{i}$, meaning numerically that $\sum_{j \in \mathcal{J}} C_{i j} v_{j}+v_{k}-\lambda v_{i} \geq \epsilon$, then it follows that $C_{i k}=C_{k i}=0$, since all components of $\mathbf{v}$ are positive. (b) If $\sum_{j \in \mathcal{J}} C_{i j} v_{j}+v_{k} \leq \lambda v_{i}$, meaning numerically that $\sum_{j \in \mathcal{J}} C_{i j} v_{j}+v_{k}-\lambda v_{i}<\epsilon$, then $C_{i k}=C_{k i}$ is allowed to assume both values 0 and 1 , and the search branches. In both cases (a) and (b), the algorithm continues recursively until the set $\mathcal{K}^{\prime}=\mathcal{K} \backslash k$ of 'unknown' entries is empty, and either a failure or a success is reported. 
After examining the $i$-th line in step (iii), the algorithm has found all possible binary matrices fulfilling the $i$-th line of the secular equation, as well as the lines previously treated. For each of these partial solutions, the reconstruction continues with the line corresponding to the next largest component of $\mathbf{v}$. Thereby, a tree is constructed which grows by another generation $t$ every time a new line of the secular equation is evaluated. The leaves of the tree at generation $t$ are the partial solutions that fulfill the so far treated $t$ lines of the secular equation. Some of the leaves of the tree 'die' in the step from generation $t-1$ to $t$, if no solution for the presently treated line of the secular equation is found that fulfills the previously treated $t-1$ lines of the secular equation as well. The search continues either until all lines of the secular equation have been treated, or until all leaves have 'died.' In the former case, at least one complete solution is found, whereas in the latter case the reconstruction stops without result. Due to the particular order of the search, the exponentially large number of possible CMs is explored in a 'greedy' way, limiting the search to the smallest possible subset by discarding wrong parts of the tree already in an early stage of the search. For most of the proteins we have studied (see below), the number of leaves in any given step did not exceed the value of $10^{4}$ for a properly chosen value of the threshold $\epsilon$.

Notice that the elements $C_{i j}=C_{j i},|i-j|>2$, for which both $v_{i}$ and $v_{j}$ vanish are not determined by the $\mathrm{PE}$. These positions belong to a set which does not interact with the set of positions contributing to the $\mathrm{PE}$, either as a completely independent structural domain, or as an isolated residue without any contact. In step (ii), we set such values of $C_{i j}=C_{j i}$ to 0 for convenience. Their actual value can be determined at a later stage, when the CM obtained through our algorithm is submitted to a procedure such as the one of Ref. [8], in order to determine the three-dimensional structure. This second step yields the three-dimensional structure, satisfying physical constraints, whose CM is most similar to the one reconstructed from the PE.

If the principal eigenvalue $\lambda$ is not known, a simple way to guess its value consists in taking the smallest non-zero $\mathrm{PE}$ component $v_{i}$, and use (i) all ratios $v_{j} / v_{i}$ with nonzero $v_{j}$ and $|i-j|>2$, (ii) all ratios $\left(v_{j}+v_{k}\right) / v_{i}$ with non-zero $v_{j}$ and $v_{k}, k>j,|i-j|>2$, and $|i-k|>2$, and (iii) all ratios $\left(v_{j}+v_{k}+v_{l}\right) / v_{i}$ with non-zero $v_{j}, v_{k}$, and $v_{l}, l>k>j,|i-j|>2,|i-k|>2$, and $|i-l|>2$ as candidate values. The first choice corresponds to assuming that position $i$ has only a single contact, the second corresponds to assuming that position $i$ has two contacts, whereas the third corresponds to assuming that position $i$ has three contacts. Values larger than 10 (for CMs on a $4.5 \AA$ threshold on the heavy atoms) are discarded. In this way, one can find a set of 'guesses' of the principal eigenvalue, which, for CMs of proteins, contains the correct value. All wrong guesses for the principal eigenvalue get quickly discarded by the algorithm, since the simulation runs into a dead end in these cases.

Another important issue concerns the choice of the numerical threshold $\epsilon$. If the threshold is too small, it may be that no solution is found, due to the numerical roundoff error on the PE components. If it is too large, the tree branches too often and the search becomes unmanageable. In our calculations, values of order $\epsilon=10^{-6}$ represented typically a good compromise. Alternatively, as we have done in this study, the value of $\epsilon$ can be automatically adjusted by letting it slowly increase, starting with a small value, until a solution is found. Note that alternatively to a threshold on the absolute error, it is possible to apply a threshold on the relative error. When doing so, for instance the equality $\sum_{j \in \mathcal{J}} C_{i j} v_{j}=\lambda v_{i}$ becomes $\left|\sum_{j \in \mathcal{J}} C_{i j} v_{j}-\lambda v_{i}\right| /\left(\lambda v_{i}\right)<\epsilon$. The latter condition is usually more appropriate if the entries $v_{i}$ are quite broadly distributed.

Results. - In order to assess the performance of the algorithm, we set out to reconstruct the 221 globular protein structures of PDBselect25 of length $N \leq 120$. We diagonalized the CMs of these 221 proteins to obtain their PE. Then, we applied to each PE the reconstruction scheme described above. In 205 cases, the algorithm yielded a unique solution, identical to the original native CM. Clearly, in these cases it is possible to recover the three-dimensional protein structure with the same accuracy achieved when starting with the native CM, for instance using the scheme of Ref. [8], with a typical root mean square displacement (RMSD) of around $2 \AA$. In the remaining 16 cases, the algorithm yielded a unique solution, yet the reconstructed CM differed from the original one in one or several missing contacts, up to $8 \%$ of the native ones, which were undetermined since the corresponding pairs of components of the PE are 0 . Nevertheless, the reconstructed CMs were very similar to the native ones, so that it is still possible to recover the three-dimensional protein structure with a high accuracy, as the RMSD was increased by $10 \%$ or less with respect to the structure obtained using the native CM. In all cases only a single CM was found and therefore the PE defines essentially in a unique way a $\mathrm{CM}$, and no false contact was contained in any of the obtained CMs.

It should be noted that the reconstruction of the $\mathrm{CMs}$ can differ considerably in computational expenses, the three most difficult cases in the present set of proteins being the CM of PDB id. 1gif $\_$A $(N=115)$, of PDB id. 1poa $(N=118)$, and of PDB id. 1bnk $\_$A $(N=120)$. For these proteins, there are many almost identical non-zero components of the $\mathrm{PE}$, so that our strategy to efficiently eliminate dead ends was not very effective, leading to an excessive branching of the search. However, even in these cases the solution of the secular equation is unique and could be found with an extensive search.

Discussion. - We have shown that the PE determines uniquely the $\mathrm{CM}$, apart from elements that correspond to pairs of positions with vanishing $\mathrm{PE}$ components and 
are hence undetermined. This is a surprising result, since it means that the remaining $N-1$ eigenvectors and eigenvalues of the CM, where $N$ is of order 100 or larger, are completely determined by the PE. It can be understood by noting that the number of CMs is large but finite: The total number of $N \times N$ symmetric binary matrices is $2^{N(N+1) / 2}$, whereas the total number of CMs representing protein structures increases with $N$ as $\exp (a N)$, since the chain connectivity introduces correlations among the contacts of neighboring residues [9]. In contrast, the number of PEs which are non-identical up to a given precision $\epsilon$ increases with decreasing $\epsilon$ as $\epsilon^{-N}$ without bound. Therefore, for sufficiently small $\epsilon$, the number of these distinct PEs is much larger than the number of CMs, so that the overwhelming majority of them do not correspond to any CM, and we can expect that those which do correspond to a CM correspond to a unique CM, as our results confirm. We note that this is true, however, only for sufficiently accurate PEs. For noisy PEs, the exact algorithm presented in this Letter is not suitable, and one has to resort to a stochastic search, such as for example a Monte Carlo scheme. Such scheme is considerably more complicated than the algorithm presented here, mainly due to the existence of CMs which do not correspond to a physically realizable structure but have a PE being almost identical to the one of the target CM. Preliminary results suggest that the reconstruction of a noisy PE might be possible when constraining the search to CMs corresponding to protein-like structures with proper secondary structure and steric interaction.

The PE only contains information on the largest connected component of the graph representing the protein structure. Nodes which are not connected with it have vanishing $\mathrm{PE}$ components, and their mutual links are undetermined. Therefore, our scheme is only able to determine the $\mathrm{CM}$ for the subset of positions which have non-zero PE components. For single-domain globular proteins, they represent the great majority of the positions in the chain, with the only exceptions of small loops or single residues completely exposed to the solvent. For non-globular proteins, whose CM is sparse and whose number of contacts per residue is hence smaller than a threshold, the connected positions are few and the method is able to yield only a portion of the CM. However, such structures are problematic in any case, since their CM does not determine a well defined threedimensional structure, and they are not thermodynamically stable in absence of other protein chains and other molecules with which they interact (there are 27 such protein structures in PDBselect25 of length $N \leq 120$ which we omitted in our study). More important difficult cases are structures constituted of several almost independent domains. For such structures, the PE components are very small outside the principal domain, although they are not zero, since there is always a small number of inter-domain contacts.

In conclusion, we have presented an exact algorithm that allows the CM of a protein structure to be reconstructed from the sole knowledge of its PE. The resulting $\mathrm{CM}$ can then be used to reconstruct the full threedimensional structure, for instance using the scheme of Ref. 8]. In this sense, the three-dimensional structure of a protein fold can be reduced ('compressed') into the PE of its CM, from which it can be recovered ('decompressed') with no or minimal information loss. We have applied the algorithm to the set of 221 globular proteins of PDBselect25 of length $N \leq 120$ and obtained in all cases a unique $\mathrm{CM}$ from the $\mathrm{PE}$. In terms of structure representation, our results show that a $\mathrm{CM}$ and its $\mathrm{PE}$ are equivalent, which leads to a significant simplification in the representation of protein folds. We anticipate that this result will create new possibilities in bioinformatics applications, in particular those involving alignments of structure to structure and of sequence to structure. Furthermore, our results have important implications on our understanding of protein evolution. We have shown in fact that the PE is correlated with the hydrophobicity profile of the amino acid sequence attaining the fold, and even more correlated with the hydrophobicity profile averaged over many sequences attaining the fold, so that protein evolution can be understood as the motion of the hydrophobicity profile around the PE of the fold [12]. Finally, since the PE is related to the contact vector 13], which it seems to be possible to predict from the protein sequence [14], a coarse prediction of the PE may be possible as well and thus the PE may become an effective tool in protein structure prediction approaches.

[1] I. Koh, V. Eyrich, M. Marti-Renom, D. Przybylski, M. Madhusudhan, N. Eswar, O. Grana, F. Pazos, A. Valencia, A. Sali, et al., Nucl. Ac. Res. 31, 3311 (2003).

[2] See http://predictioncenter.llnl.gov.

[3] S. Lifson and C. Sander, Nature 282, 109 (1979).

[4] P. Fariselli, O. Olmea, A. Valencia, and R. Casadio, Prot. Eng. 14, 835 (2001).

[5] L. Holm and C. Sander, Proteins 19, 256 (1994).

[6] N. Kannan and S. Vishveshwara, J. Mol. Biol. 292, 441 (1999).

[7] N. Kannan and S. Vishveshwara, Prot. Eng. 13, 753 (2000).

[8] M. Vendruscolo, E. Kussell, and E. Domany, Fold. \& Des. 2, 295 (1997).

[9] M. Vendruscolo, B. Subramanian, I. Kanter, E. Domany, and J. L. Lebowitz, Phys. Rev. E 59, 977 (1999).

[10] J. England and E. Shakhnovich, Phys. Rev. Lett. 90, 218101 (2003).

[11] B. Bollobas, Modern graph theory (Springer Verlag, Berlin, 1998).

[12] U. Bastolla, M. Porto, H. Roman, and M. Vendruscolo, submitted.

[13] A. Kabakcioglu, I. Kanter, M. Vendruscolo, and E. Domany, Phys. Rev. E 65, 041904 (2002).

[14] G. Pollastri, P. Baldi, P. Fariselli, and R. Casadio, Proteins $\mathbf{4 7}, 142$ (2002). 